\title{
Does Chlorhexidine Bathing in Adult Intensive Care Units Reduce Blood Culture Contamination? A Pragmatic Cluster-Randomized Trial
}

\author{
Edward J. Septimus, MD; ${ }^{1,2}$ Mary K. Hayden, MD; ${ }^{3}$ Ken Kleinman, ScD; ${ }^{4}$ Taliser R. Avery, MS; ${ }^{4}$ Julia Moody, MS; ${ }^{1}$ \\ Robert A. Weinstein, MD; Jason Hickok, MBA, RN; ${ }^{1}$ Julie Lankiewicz, MPH; ${ }^{4}$ Adrijana Gombosev, BS; ${ }^{6}$ \\ Katherine Haffenreffer, BS; ${ }^{4}$ Rebecca E. Kaganov, BA; ${ }^{4}$ John A. Jernigan, MD, MS; ${ }^{7}$ \\ Jonathan B. Perlin, MD, PhD; ${ }^{1}$ Richard Platt, MD, MS; ${ }^{4}$ Susan S. Huang, MD, MPH ${ }^{6}$
}

\begin{abstract}
ов ВестіVE. To determine rates of blood culture contamination comparing 3 strategies to prevent intensive care unit (ICU) infections: screening and isolation, targeted decolonization, and universal decolonization.

DESIGN. Pragmatic cluster-randomized trial.

SETTING. Forty-three hospitals with 74 ICUs; 42 of 43 were community hospitals.

PATIENTS. Patients admitted to adult ICUs from July 1, 2009, to September 30, 2011.

METHODS. After a 6-month baseline period, hospitals were randomly assigned to 1 of 3 strategies, with all participating adult ICUs in a given hospital assigned to the same strategy. Arm 1 implemented methicillin-resistant Staphylococcus aureus (MRSA) nares screening and isolation, arm 2 targeted decolonization (screening, isolation, and decolonization of MRSA carriers), and arm 3 conducted no screening but universal decolonization of all patients with mupirocin and chlorhexidine (CHG) bathing. Blood culture contamination rates in the intervention period were compared to the baseline period across all 3 arms.
\end{abstract}

RESUlTS. During the 6-month baseline period, 7,926 blood cultures were collected from 3,399 unique patients: 1,099 sets in arm 1, 928 in arm 2, and 1,372 in arm 3. During the 18-month intervention period, 22,761 blood cultures were collected from 9,878 unique patients: 3,055 sets in arm 1, 3,213 in arm 2, and 3,610 in arm 3. Among all individual draws, for arms 1, 2, and 3, the contamination rates were $4.1 \%, 3.9 \%$, and $3.8 \%$ for the baseline period and $3.3 \%, 3.2 \%$, and $2.4 \%$ for the intervention period, respectively. When we evaluated sets of blood cultures rather than individual draws, the contamination rate in arm 1 (screening and isolation) was $9.8 \%(N=108$ sets) in the baseline period and 7.5\% $(N=228)$ in the intervention period. For arm 2 (targeted decolonization), the baseline rate was $8.4 \%(N=$ 78) compared to $7.5 \%(N=241)$ in the intervention period. Arm 3 (universal decolonization) had the greatest decrease in contamination rate, with a decrease from $8.7 \%(N=119)$ contaminated blood cultures during the baseline period to $5.1 \%(N=184)$ during the intervention period. Logistic regression models demonstrated a significant difference across the arms when comparing the reduction in contamination between baseline and intervention periods in both unadjusted $(P=.02)$ and adjusted $(P=.02)$ analyses. Arm 3 resulted in the greatest reduction in blood culture contamination rates, with an unadjusted odds ratio (OR) of 0.56 ( $95 \%$ confidence interval $[\mathrm{CI}]$, $0.044-0.71)$ and an adjusted OR of 0.55 (95\% CI, 0.43-0.71).

CONCLusion. In this large cluster-randomized trial, we demonstrated that universal decolonization with CHG bathing resulted in a significant reduction in blood culture contamination.

Infect Control Hosp Epidemiol 2014;35(S3):S17-S22

Blood cultures are a critical tool to diagnose bacteremia and guide antimicrobial therapy, especially with the increasing threat of multidrug-resistant organisms (MDROs). However, contamination of blood cultures is still a common problem and may represent up to half of all positive blood cultures. ${ }^{1,2}$ The Clinical and Laboratory Standards Institute recommends that each facility maintain a contamination rate less than $3 \% .^{3}$ The American Society for Microbiology's and College of

Affiliations: 1. Hospital Corporation of America, Nashville, Tennessee; 2. Texas A\&M Health Science Center, College of Medicine, Texas A\&M University, Houston, Texas; 3. Rush University Medical Center, Chicago, Illinois; 4. Harvard Medical School and Harvard Pilgrim Health Care Institute, Harvard University, Boston, Massachusetts; 5. Cook County Health and Hospitals System, Chicago, Illinois; 6. University of California Irvine School of Medicine, Orange, California; 7. Centers for Disease Control and Prevention (CDC), Atlanta, Georgia, and for the Agency for Healthcare Research and Quality Developing Evidence to Inform Decisions about Effectiveness Network and Healthcare-Associated Infections Program and the CDC Prevention Epicenters Program.

Received March 17, 2014; accepted April 4, 2014; electronically published September 15, 2014.

(C) 2014 by The University of Chicago. All rights reserved. 0899-823X/2014/35S3-0004\$15.00. DOI: $10.1086 / 677822$ 
American Pathologists' benchmark for contaminated blood cultures hospital wide is between $2.5 \%$ and $3 \%{ }^{4,5}$ In recent studies the overall blood culture contamination rate in intensive care unit (ICU) populations ranged from $4 \%$ to $5.5 \% .{ }^{6.7}$ "Contamination" in these reports is defined as the number of contaminated blood cultures divided by the total number of blood culture draws multiplied by 100. Contamination can lead to unnecessary antimicrobial therapy, unnecessary removal of central lines, unnecessary testing, increased length of stay, and increased cost. Bates et $\mathrm{al}^{8}$ estimated the cost of contaminated blood cultures at $\$ 4,500$ per episode. Patients with contaminated blood cultures are just as likely to receive antimicrobial therapy as patients with true bacteremia. ${ }^{9}$ It has been reported that up to half of patients with false-positive blood cultures for coagulase-negative staphylococci were treated with antibiotics, usually vancomycin, with an estimated additional cost of approximately $\$ 1,000$ per patient. ${ }^{10}$ This association between contaminated blood cultures and unnecessary antibiotic use, additional laboratory tests, and increased hospital length of stay and excess costs has been confirmed in subsequent studies, with costs as high as $\$ 10,000$. $^{11,12}$

Contamination of percutaneous blood cultures is thought to be due to the introduction of organisms from the skin of the patient into the collected sample. ${ }^{13}$ Inadequate preparation of the skin is thought to be the most common cause of blood culture contamination. ${ }^{14}$ This is supported by surveys of the most common organisms in contaminated blood cultures, which represent organisms that are known to be present on the skin of hospitalized patients. The most common contaminant is coagulase-negative staphylococci, which accounts for approximately $75 \%$ of contaminated blood cultures, followed by Propionibacterium sp., Micrococcus sp., Corynebacterium sp., Bacillus sp. (not Bacillus anthracis), Micrococcus sp., viridans streptococci, and $\gamma$-hemolytic streptococci (not Enterococcus sp.). ${ }^{4,9,15}$ Accordingly, interventions that have been studied to reduce contamination are those that could reduce skin bacterial load or reduce the likelihood of inadvertent introduction of skin contaminants into the sample. These include disinfection methods for skin preparation, culture bottle preparation, needle exchange for bottle inoculation, limiting the use of blood drawn from intravenous lines, and the use of dedicated phlebotomy teams. ${ }^{4}$ Recently, several studies have suggested that chlorhexidine ( $\mathrm{CHG}$ ) bathing of patients in the ICU may reduce blood culture contamination rates. ${ }^{16-18}$

In our previous publication, ${ }^{19}$ we reported that universal decolonization was more effective than targeted decolonization or screening and isolation in reducing methicillin-resistant Staphylococcus aureus (MRSA) clinical cultures and bloodstream infections from any pathogen. In this study, we investigated whether these 3 strategies to prevent ICU infections would have an effect on the rates of blood culture contamination.

\section{METHODS}

\section{Study Design}

The Randomized Evaluation of Decolonization vs Universal Clearance to Eradicate MRSA (REDUCE MRSA) trial was a 3-arm cluster-randomized trial of hospitals in the Hospital Corporation of America (HCA) system. This study compared 3 strategies to decrease MRSA infections in adult ICUs. Elements of the trial design have been previously described. ${ }^{17}$ The strategies were limited to the adult ICU and included:

- Arm 1: Screening and isolation: Patients received bilateral nares screening for MRSA upon ICU admission. Contact precautions were employed for patients with a known history of MRSA (either colonization or infection) or a current culture or screening test positive for MRSA. This arm was considered standard of care at the time and in practice since $2007 . .^{18}$

- Arm 2: Targeted decolonization: At ICU admission, patients received MRSA screening and contact precautions similar to arm 1. Patients with known MRSA colonization received decolonization with twice daily intranasal $2 \%$ mupirocin ointment and daily $2 \%$ CHG cloth baths for 5 days.

- Arm 3: Universal decolonization: There was no screening for MRSA at ICU admission. Contact precautions similar to arm 1 were employed. All ICU patients received twice daily intranasal mupirocin ointment for 5 days plus daily $2 \%$ CHG cloth baths for the entire duration of their ICU stay.

This study consisted of a 6-month baseline period from July 1 to December 31, 2009; a phase-in period from January 1 to April 7, 2010; and an 18-month intervention period from April 8, 2010, to September 30, 2011. This study was approved by the Harvard Pilgrim Health Care institutional review board.

\section{Determination of Blood Culture Contamination}

For descriptive purposes, we provide the percent of all blood culture draws either by a percutaneous (direct skin puncture) or from an existing intravascular catheter that contained a skin commensal consistent with contamination. The performance of an individual blood culture commonly involves collecting a volume of blood either via venipuncture or from an intravascular catheter and distributing it into 1 or more bottles (eg, an aerobic and anaerobic bottle). However, in keeping with clinical application, all analyses were performed at the level of blood culture sets unless otherwise specified. Blood culture sets were eligible for the determination of contamination if at least 2 ICU-attributed blood cultures were drawn within 2 calendar days of one another. Thus, a single blood culture draw within a 2-day period was excluded from evaluation. Two or more blood cultures during that time window constituted a single set. Only the first eligible set per patient was evaluated. Blood cultures were deemed attrib- 
TABLE 1. Patient Characteristics by Strategies to Reduce Blood Culture Contamination in Intensive Care Units

\begin{tabular}{|c|c|c|c|c|c|c|c|c|}
\hline \multirow[b]{2}{*}{ Variable } & \multicolumn{4}{|c|}{ Baseline, 6 months, $N=3,399$} & \multicolumn{4}{|c|}{ Intervention, 18 months, $N=9,878$} \\
\hline & Arm 1 & Arm 2 & Arm 3 & $P$ & Arm 1 & Arm 2 & Arm 3 & $P$ \\
\hline \multicolumn{8}{|l|}{ Admissions with ICU stay and } & $\ldots$ \\
\hline Draws per patient, median (IQR) & $2(0)$ & $2(0)$ & $2(0)$ & .6 & $2(0)$ & $2(0)$ & $2(0)$ & .5 \\
\hline $\begin{array}{l}\text { Length of hospital stay, days, } \\
\text { median (IQR) }\end{array}$ & $15(15)$ & $14(15.5)$ & $15(16)$ & .6 & $14(13)$ & $14(14)$ & $14(14)$ & .9 \\
\hline \multicolumn{9}{|l|}{ Length of ICU stay, days, me- } \\
\hline Age, years, median (IQR) & $64(23)$ & $65(22)$ & $63(26)$ & .5 & $64(23)$ & $65(24)$ & $63(24)$ & .5 \\
\hline Female & 43.1 & 44.3 & 45.6 & .6 & 42.3 & 45.3 & 44.5 & .4 \\
\hline Race & & & & 1.0 & & & & 1.0 \\
\hline White & 73.0 & 75.1 & 67.1 & $\ldots$ & 73.9 & 74.5 & 66.9 & $\ldots$ \\
\hline Black & 14.9 & 12.8 & 10.6 & $\ldots$ & 14.6 & 12.9 & 10.0 & $\ldots$ \\
\hline Hispanic & 4.6 & 8.5 & 16.9 & $\ldots$ & 4.7 & 8.9 & 18.8 & $\ldots$ \\
\hline Asian & 2.7 & 1.0 & 1.1 & $\ldots$ & 2.7 & 1.1 & 0.6 & $\ldots$ \\
\hline Other & 2.3 & 1.3 & 2.5 & $\ldots$ & 2.5 & 1.0 & 2.4 & $\ldots$ \\
\hline Unknown & 2.5 & 1.3 & 1.9 & $\ldots$ & 1.6 & 1.6 & 1.3 & $\ldots$ \\
\hline Insurance & & & & 1.0 & & & & 1.0 \\
\hline Medicare & 56.1 & 60.3 & 56.9 & $\ldots$ & 58.1 & 60.9 & 57.1 & $\ldots$ \\
\hline Commercial & 23.0 & 19.4 & 19.3 & $\ldots$ & 20.7 & 18.1 & 18.9 & $\ldots$ \\
\hline Medicaid & 11.0 & 13.1 & 13.7 & $\ldots$ & 10.7 & 11.0 & 11.5 & $\ldots$ \\
\hline Self-pay & 5.9 & 4.3 & 5.2 & $\ldots$ & 6.3 & 6.5 & 6.4 & $\ldots$ \\
\hline Free care & 2.1 & 2.3 & 2.8 & $\ldots$ & 1.5 & 2.0 & 3.4 & $\ldots$ \\
\hline Other & 1.8 & 0.5 & 1.5 & $\ldots$ & 2.6 & 1.3 & 2.2 & $\ldots$ \\
\hline Unknown & 0.0 & 0.0 & 0.5 & $\ldots$ & 0.1 & 0.1 & 0.5 & $\ldots$ \\
\hline \multicolumn{9}{|l|}{ Comorbidities } \\
\hline COPD & 29.7 & 30.4 & 28.1 & .9 & 31.3 & 30.9 & 29.0 & .9 \\
\hline Diabetes & 29.8 & 32.8 & 30.1 & .7 & 30.4 & 32.1 & 29.9 & .6 \\
\hline Congestive heart failure & 30.5 & 34.4 & 28.1 & .4 & 30.7 & 31.8 & 29.2 & .9 \\
\hline Renal failure & 22.9 & 25.4 & 24.1 & .8 & 25.0 & 26.8 & 24.4 & .8 \\
\hline Myocardial infarction & 83.7 & 81.3 & 83.7 & .5 & 83.5 & 83.1 & 83.4 & 1.0 \\
\hline Cerebrovascular disease & 15.6 & 17.7 & 17.6 & .7 & 17.0 & 14.3 & 18.0 & .3 \\
\hline Peripheral vascular disease & 9.3 & 10.1 & 10.6 & .5 & 8.6 & 10.1 & 11.0 & .1 \\
\hline Cancer & 10.3 & 11.1 & 10.2 & .8 & 9.2 & 10.4 & 11.7 & .3 \\
\hline Hemiplegia/paraplegia & 6.6 & 7.0 & 7.0 & .9 & 6.8 & 6.1 & 7.1 & .9 \\
\hline Liver failure & 6.8 & 9.2 & 6.0 & 1.0 & 4.1 & 4.7 & 3.6 & .4 \\
\hline Peptic ulcer disease & 4.0 & 4.5 & 3.4 & .7 & 3.4 & 3.4 & 3.3 & 1.0 \\
\hline Rheumatologic disease & 1.7 & 3.0 & 3.1 & .1 & 2.2 & 2.6 & 3.5 & .03 \\
\hline Dementia & 1.7 & 1.7 & 2.6 & .3 & 2.3 & 2.3 & 2.5 & .6 \\
\hline AIDS & 0.7 & 0.5 & 0.9 & .8 & 1.4 & 0.7 & 0.8 & .03 \\
\hline Surgery during admission & 42.9 & 42.1 & 48.3 & .9 & 40.0 & 41.4 & 47.2 & .5 \\
\hline
\end{tabular}

NOTE. All data shown are percentages, unless otherwise indicated. COPD, chronic obstructive pulmonary disease; ICU, intensive care unit; IQR, interquartile range.

utable to the ICU if the draws occurred more than 1 day into the ICU stay through the day of ICU discharge. This attribution window was selected to allow for the first CHG bath to be given in the ICU. We were unable to determine accurately which blood cultures were drawn by venipuncture or from an existing line.

Among eligible blood culture sets, contamination was defined as having 1 or more of the following pathogens isolated from only 1 blood culture within the set: coagulase-negative Staphylococcus sp., Lactobacillus sp., Propionibacterium acnes, Corynebacterium sp., Bacillus sp. (not B. anthracis), Micro- coccus sp., viridans streptococci, and $\gamma$-hemolytic streptococci (not Enterococcus sp.). Our analysis focuses on the proportion of eligible blood culture sets that had a contamination event across all 3 study arms.

\section{Statistical Analysis}

Census and microbiologic information were obtained from the HCA centralized clinical electronic data warehouse. The HCA system uses a single electronic health record (EHR) platform including the microbiology module for test orders 
TABLE 2. Results from Logistic Regression Models for Blood Culture Contamination

\begin{tabular}{|c|c|c|c|c|}
\hline & Arm 1 & Arm 2 & Arm 3 & Overall trial \\
\hline & OR $(95 \% \mathrm{CI})$ & OR $(95 \% \mathrm{CI})$ & OR $(95 \% \mathrm{CI})$ & $P$ \\
\hline As randomized, unadjusted & $0.74(0.58-0.94)$ & $0.94(0.72-1.23)$ & $0.56(0.44-0.71)$ & .02 \\
\hline As randomized, adjusted by no. of draws & $0.74(0.58-0.95)$ & $0.93(0.71-1.23)$ & $0.56(0.44-0.71)$ & .02 \\
\hline $\begin{array}{l}\text { As randomized, adjusted by no. of draws, age, } \\
\text { sex, race, payer, surgery, and comorbidities }\end{array}$ & $0.73(0.57-0.94)$ & $0.93(0.7-1.22)$ & $0.55(0.43-0.71)$ & .02 \\
\hline As treated, unadjusted & $0.74(0.58-0.94)$ & $0.93(0.71-1.22)$ & $0.56(0.44-0.71)$ & .02 \\
\hline Randomization to all 3 arms, unadjusted & $0.75(0.58-0.96)$ & $0.9(0.68-1.19)$ & $0.56(0.44-0.71)$ & .03 \\
\hline Accounting for randomization strata, unadjusted & $0.73(0.58-0.94)$ & $0.94(0.72-1.23)$ & $0.56(0.44-0.71)$ & .02 \\
\hline Weekday draws (entire set collected on weekday) & $0.77(0.57-1.05)$ & $0.82(0.6-1.13)$ & $0.56(0.41-0.76)$ & .18 \\
\hline Weekend draws (any in set collected on weekend) & $0.66(0.44-0.99)$ & $1.2(0.72-1.98)$ & $0.56(0.39-0.83)$ & .06 \\
\hline Day draws (entire set collected 5 am-4:49 pm) & $0.71(0.5-1.02)$ & $0.97(0.68-1.39)$ & $0.64(0.46-0.9)$ & .24 \\
\hline Night draws (any in set collected 5 pm-4:59 am) & $0.79(0.56-1.11)$ & $0.9(0.59-1.37)$ & $0.44(0.31-0.62)$ & .01 \\
\hline
\end{tabular}

NOTE. $P$ values in the pairwise analysis were as follows: $P=.19$ for the comparison of arm 2 with arm $1 ; P=.11$ for the comparison of arm 3 with arm 1; and $P=.005$ for the comparison of arm 3 with arm 2 .

and resulting. The contamination rate was calculated as a percentage of eligible blood culture sets.

We used generalized linear mixed models to account for the cluster-randomized design of the trial. In that context, we used logistic regression to assess the treatment arm, period, and arm-by-period interaction effect. The 2-degree-of-freedom test assessing the interaction addresses the null hypothesis that the change from baseline to intervention period was the same in each arm. Sensitivity analysis included multivariable covariate-adjusted models that accounted for age, sex, race, insurance type, coexisting conditions, and surgery during the hospital stay. Analyses were performed with use of Statistical Analysis System (SAS) software, version 9.3 (SAS Institute).

\section{RESULTS}

Patient characteristics were similar across all groups and between baseline and intervention periods (Table 1). During the 6-month baseline period, 7,926 blood cultures were collected from 3,399 unique patients: 1,099 sets in arm 1,928 in arm 2, and 1,372 in arm 3. During the 18-month intervention period, 22,761 blood cultures were collected from 9,878 unique patients: 3,055 sets in arm 1, 3,213 in arm 2, and 3,610 in arm 3. Among all individual draws, for arms 1, 2 , and 3 , the contamination rates were $4.1 \%, 3.9 \%$, and $3.8 \%$ for the baseline period and $3.3 \%, 3.2 \%$, and $2.4 \%$ for the intervention period, respectively.

When we evaluated sets of blood cultures rather than individual draws, the contamination rate in arm 1 (screening and isolation) was $9.8 \%(N=108$ sets $)$ in the baseline period and $7.5 \%(N=228)$ in the intervention period. For arm 2 (targeted decolonization), the baseline rate was $8.4 \%(N=$ $78)$ compared to $7.5 \%(N=241)$ in the intervention period. Arm 3 (universal decolonization) had the greatest decrease in contamination rate, with a decrease from $8.7 \%(N=119)$ contaminated blood cultures during the baseline period to $5.1 \%(N=184)$ during the intervention period.
Logistic regression models for contaminated sets (Table 2) demonstrated a significant difference across the arms when comparing the reduction in contamination between baseline and intervention periods in both unadjusted $(P=.02)$ and adjusted $(P=.02)$ analyses. All arms showed a reduction in contamination between the intervention period and the baseline period, but universal decolonization resulted in the greatest reduction in blood culture contamination rates, with an unadjusted odds ratio (OR) of 0.56 (95\% confidence interval $[\mathrm{CI}], 0.044-0.71)$ and an adjusted OR of $0.55(95 \%$ $\mathrm{CI}, 0.43-0.71) . P$ values in the pairwise analysis were as follows: $P=.19$ for the comparison of arm 2 with arm $1, P=$ .11 for the comparison of arm 3 with arm 1 , and $P=.005$ for the comparison of arm 3 with arm 2. Based on the ORs, universal decolonization avoided an additional 26.8 contaminated blood culture sets per 1,000 admissions compared to arm 2 and an additional 12.2 contaminated blood culture sets per 1,000 admissions compared to arm 1 . The most common organism associated with contamination was coagulasenegative Staphylococcus sp. (85.0\%), followed by Streptococcus sp. (6.4\%) and Bacillus sp. (3.0\%; Table 3).

\section{I S C USS I ON}

Contamination of blood cultures can alter the course of a patient's treatment, resulting in outcomes such as inappropriate antibiotic use, longer hospital stays, or increased cost. Evidence suggests that the rate of blood culture contamination could potentially be influenced by interventions that decrease skin bacterial load, such as CHG bathing.

Bleasdale et $\mathrm{al}^{16}$ examined the effectiveness of $\mathrm{CHG}$ bathing to reduce central line-associated bloodstream infections (CLABSIs) in a 2-ICU crossover study. They reported a significant reduction in primary BSIs and a nonsignificant reduction in the incidence of blood culture contamination from 4.3 to 1.8 per 1,000 patient-days. Popovich et $\mathrm{al}^{17,18}$ have reported significant declines in blood culture contamination 
TABLE 3. Blood Culture Contamination Pathogens by Genus Group

\begin{tabular}{|c|c|c|c|c|c|c|c|}
\hline & \multicolumn{3}{|c|}{ Baseline } & \multicolumn{3}{|c|}{ Intervention } & \multirow[b]{2}{*}{ Total } \\
\hline & Arm 1 & Arm 2 & Arm 3 & Arm 1 & Arm 2 & Arm 3 & \\
\hline Aerococcus sp. & 0 & 0 & 0 & $1(0.3)$ & 0 & 0 & $1(0.1)$ \\
\hline Bacillus sp. (not anthracis) & $5(3.1)$ & $4(3.6)$ & $6(4.1)$ & $9(2.7)$ & $8(2.3)$ & $8(3.5)$ & $40(3)$ \\
\hline Corynebacterium sp. & $2(1.2)$ & $3(2.7)$ & $3(2.1)$ & $8(2.4)$ & $9(2.6)$ & $5(2.2)$ & $30(2.3)$ \\
\hline Diphtheroids & $3(1.9)$ & 0 & $1(0.7)$ & $5(1.5)$ & $2(0.6)$ & 0 & $11(0.8)$ \\
\hline Lactobacillus sp. & 0 & 0 & 0 & 0 & $1(0.3)$ & $1(0.4)$ & $2(0.2)$ \\
\hline Micrococus sp. & $1(0.6)$ & 0 & $2(1.4)$ & $4(1.2)$ & $3(0.9)$ & $3(1.3)$ & $13(1)$ \\
\hline Peptostreptococcus sp. & 0 & 0 & $1(0.7)$ & $2(0.6)$ & 0 & $2(0.9)$ & $5(0.4)$ \\
\hline Propionibacterium $\mathrm{sp}$. & 0 & 0 & $2(1.4)$ & $3(0.9)$ & $4(1.2)$ & 0 & $9(0.7)$ \\
\hline Saccharomyces sp. & $2(1.2)$ & 0 & 0 & $1(0.3)$ & 0 & 0 & $3(0.2)$ \\
\hline $\begin{array}{l}\text { Staphylococcus, coagulase- } \\
\text { negative species }\end{array}$ & $136(84)$ & $93(83)$ & $128(88.3)$ & $274(83.3)$ & $293(85.7)$ & $196(86)$ & $1,120(85)$ \\
\hline Streptococcus sp. & $13(8)$ & $12(10.7)$ & $2(1.4)$ & $22(6.7)$ & $22(6.4)$ & $13(5.7)$ & $84(6.4)$ \\
\hline Total pathogens & 162 & 112 & 145 & 329 & 342 & 228 & 1,318 \\
\hline
\end{tabular}

NOTE. All data are no. (\%). Multiple pathogens per specimen were allowed.

* Viridans group and gamma hemolytic Streptococcus, not Enterococcus sp.

rates in both medical ICUs (6.99 to 4.1 per 1,000 patientdays) and surgical ICUs (5.97 to 2.41 per 1,000 patient-days).

In this large cluster-randomized trial, we demonstrated that universal decolonization with CHG bathing resulted in a significant reduction in blood culture contamination. Of interest the $45 \%$ reduction in blood culture contamination with universal decolonization is virtually identical to the $44 \%$ reduction of all cause bloodstream infections previously reported. ${ }^{19}$

In this study, we observed a decrease in blood culture contamination in all arms of the study, suggesting a secular trend despite the comprehensive efforts to prevent competing interventions during the trial. ${ }^{19}$ While we inquired monthly about competing interventions and participating hospitals were instructed to bring any product or practice changes before the study steering committee, it is possible that national attention to skin or line connector preparation prior to phlebotomy, national efforts to support drawing blood by venipuncture whenever possible, and the concept of dedicated phlebotomy teams may have had a secular impact.

Almost all HCA facilities track blood culture contamination rates as a quality indicator, with appropriate action taken when rates are determined to be greater than $3 \%$ after blood culture draws. This included reeducation along retraining healthcare professionals' skills on specimen collection and limiting use of blood draws from intravenous lines. Also, to address national guidance for best practice, HCA launched a campaign as part of a patient safety initiative to reduce CLABSIs, emphasizing maintenance of lines including "scrubbing the hub" and standardizing connectors to meet certain design and safety criteria, making it easier to adequately disinfect the hub. This initiative was equally applied across all 3 arms. This initiative, along with tracking contamination rates, probably explains the secular trend noted in this analysis.

We believe that the benefit attributable to universal de- colonization (arm 3) relates to reducing patient bioburden by reducing skin colonization ${ }^{2 n}$ since inadequate skin preparation is thought to be the most common cause of blood culture contamination. ${ }^{4,13}$ In addition, our protocol included not only cleaning of the skin with $2 \%$ CHG cloths but also wiping of the proximal 6 inches of the line, including the connectors and hubs, with $2 \%$ CHG cloths. Coagulase-negative Staphylococcus sp. was the most common contaminant in our trial, consistent with other publications. ${ }^{4,9,15}$

The strength of our study was the large size and rigorous design as a pragmatic comparative effectiveness trial implemented primarily through the hospital processes. This design was chosen so that the implementation could be generalized to the broadest set of hospitals with available resources. Our study, however, had several limitations. Since microbiologic data were captured through our early clinical data warehouse, we were not then able to capture clinical signs or symptoms that could be associated with a clinical infection. In addition we were unable to account for the method of blood draw, including the method of skin cleaning and whether blood cultures were taken peripherally or from an existing line. However, such differences across groups are largely accounted for by comparing the outcome rate in each hospital with that hospital's baseline rate, providing reassurance that the benefit is attributable to decolonization rather than to baseline variation in case-mix or clinical practices across groups.

In conclusion, our study, along with other studies, clearly demonstrated that $\mathrm{CHG}$ bathing has a role in decreasing blood culture contamination rates. As this study showed, interventions targeted at different outcomes, such as the reduction of bloodstream infections, can also drive improvements in the rate of blood culture contaminations. Reduction of blood culture contamination can improve the quality of blood culture results and can prevent unnecessary antibiotics, decrease cost, and decrease length of stay. 


\section{ACKNOWLEDGMENTS}

Financial support. This project was funded by the Healthcare-Associated Infections Program under contracts HHSA290201000008I and HHSA29032007T from the Agency for Healthcare Research and Quality (AHRQ), US Department of Health and Human Services, as part of the Developing Evidence to Inform Decisions about Effectiveness program, and the Centers for Disease Control and Prevention (CDC) Prevention Epicenters Program (1U01 CI000344 to R.P.). The REDUCE MRSA study received approval from the Harvard Pilgrim Health Care institutional review board.

Potential conflicts of interest. E.J.S., M.K.H., K.K., T.R.A., J.M., R.A.W., J.H., J.L., A.G., K.H., R.E.K., J.A.J., J.B.P., and S.S.H. are conducting a trial of hospitals that are receiving product contribution from Sage Products and Molnlycke. This disclosure arose after the conduct and analysis of both the original REDUCE MRSA trial and this manuscript. E.J.S. reports receiving consulting fees from $3 \mathrm{M}$ and lecture fees from Sage Products. M.K.H. reports conducting other research involving contributed product from Sage Products. The authors of this article are responsible for its content. All authors submitted the ICMJE Form for Disclosure of Potential Conflicts of Interest, and the conflicts that the editors consider relevant to this article are disclosed here.

Address correspondence to Edward J. Septimus, MD, 4257 Albans Street, Houston, TX 77005 (edward.septimus@hcahealthcare.com).

The findings and conclusions in this report are those of the authors and do not necessarily represent the official position of the Centers for Disease Control and Prevention. The contents of this publication does not necessarily reflect the views or policies of the US Department of Health and Human Services, nor does mention of trade names, commercial products, or organizations imply endorsement by the US government. The authors assume full responsibility of the accuracy and completeness of the ideas presented.

\section{REFERENCES}

1. Weinbaum FI, Lavie S, Danek M, Sixsmith D, Heinrich GF, Mill SS. Doing it right the first time: quality improvement and the contaminant blood culture. J Clin Microbiol 1997;35(3):563-565.

2. Weinstein MP. Blood culture contamination: persisting problems and partial progress. J Clin Microbiol 2003;41:2275-2278.

3. Clinical and Laboratory Standards Institute (CLSI). Principles and Procedures for Blood Cultures: Approved Guideline. Wayne, PA: CLSI; 2007. CLSI document M47-A.

4. Hall KK, Lyman JA. Updated review of blood culture contamination. Clin Microbiol Rev 2006;19(4):788-802.

5. Bekeris LG, Tworek JA, Walsh MK, et al. Trends in blood culture contamination: a College of American Pathologists Q-Tracks study of 356 institutions. Arch Pathol Lab Med 2005;129:12221225.

6. Stohl S, Benenson S, Sviri S, et al. Blood culture at central line insertion in the intensive care unit: comparison with peripheral venipuncture. J Clin Microbiol 201 1;49:2398-2403.

7. Darby JM, Linden P, Pasculle W, Saul M. Utilization and di- agnostic yield of blood cultures in a surgical intensive care unit. Crit Care Med 1997;25:989-994.

8. Bates DW, Goldman L, Lee TH. Contaminant blood cultures and resource utilization: the true consequences of false-positive results. JAMA 1991;265:365-369.

9. Pien BC, Sundaram P, Raoof N, et al. The clinical and prognostic importance of positive blood cultures in adults. Am J Med 2010; 123(9):819-828.

10. Souvenir D, Anderson DE Jr, Palpant S, et al. Blood cultures positive for coagulase-negative staphylococci: antisepsis, pseudobacteremia, and therapy of patients. J Clin Microbiol 1998;36: 1923-1926.

11. Gander RM, Byrd L, DeCrescenzo M, et al. Impact of blood cultures drawn by phlebotomy on contamination rates and health care costs in a hospital emergency department. J Cin Microbiol 2009;47:1021-1024.

12. Alahmadi YM, Aldeyab MA, McElnay IC, et al. Clinical and economic impact of contaminated blood cultures within the hospital setting. J Hosp Infect 2011;77(3):233-236.

13. Viagappan M, Kelsey MC. The origin of coagulase-negative staphylococci isolated from blood cultures. J Hosp Infect 1995; 30:217-223.

14. Mylotte JM, Tayara A. Blood cultures: clinical aspects and controversies. Eur J Clin Microbiol Infect 2000;19:157-163.

15. Washer LL, Chenoweth C, Kim H-W, et al. Blood culture contamination: a randomized trial evaluating the comparative effectiveness of 3 skin antiseptic interventions. Infect Control Hosp Epidemiol 2013;34:15-21.

16. Bleasdale SC, Trick WE, Gonzalez IM, et al. Effectiveness of chlorhexidene bathing to reduce catheter-associated bloodstream infections in medical intensive care unit patients. Arch Intern Med 2007;167:2073-2079.

17. Popovich KJ, Hota B, Hayes BA, et al. Effectiveness of routine patient cleansing with chlorhexidene gluconate for infection prevention in the medical intensive care unit. Infect Control Hosp Epidemiol 2009;30:959-963.

18. Popovich KJ, Hota B, Hayes BA, et al. Daily skin cleansing with chlorhexidene did not reduce the rate of central-line associated bloodstream infection in a surgical intensive care unit. Intensiv Care Med 2010;36:854-858.

19. Huang SS, Septimus E, Kleinman K, et al. Targeted versus universal decolonization to prevent ICU infection. $N$ Engl J Med 2013;368:2255-2265.

20. Popovich KJ, Lyles R, Hayes BA, et al. Relationship between chlorhexidine gluconate skin concentration and microbial density on the skin of critically ill patients bathed daily with chlorhexidine gluconate. Infect Control Hosp Epidemiol 2012;33:889896. 